\title{
Effects of thermal and exozodiacal background on space telescope observations of exoEarths
}

\section{Carl T. Coker, Ji Wang, Stuart Shaklan}

Carl T. Coker, Ji Wang, Stuart Shaklan, "Effects of thermal and exozodiacal background on space telescope observations of exoEarths," Proc. SPIE 10698, Space Telescopes and Instrumentation 2018: Optical, Infrared, and Millimeter Wave, 106985G (21 August 2018); doi: 10.1117/12.2313695

SPIE Event: SPIE Astronomical Telescopes + Instrumentation, 2018, Austin, Texas, United States 


\title{
Effects of thermal and exozodiacal background on space telescope observations of exoEarths
}

\author{
Carl T. Coker ${ }^{\mathrm{a}, \mathrm{b}}$, Ji Wang ${ }^{\mathrm{c}}$, and Stuart Shaklan ${ }^{\mathrm{b}}$

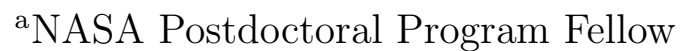 \\ ${ }^{\mathrm{b}}$ Jet Propulsion Laboratory, California Institute of Technology, 4800 Oak Grove Drive, \\ Pasadena, CA 91109 \\ ${ }^{\mathrm{c}}$ California Institute of Technology, 1200 E California Blvd, Pasadena, CA 91125
}

\begin{abstract}
One of the major goals of the exoplanet community in the coming decades is to detect Earth-like exoplanets (exoEarths) and look for biomarkers in their atmospheres. High-dispersion coronagraphy (HDC) may allow detection and characterization to be done simultaneously, as well as relax the starlight suppression requirements of the telescope and coronagraph. However, similar to other direct imaging techniques, HDC faces challenging thermal and/or exozodiacal background levels. In this paper, we present simulations of coronagraphic observations using a variety of space telescope apertures ranging in diameter from 1 to $15 \mathrm{~m}$, specifically incorporating thermal and exozodiacal background. We investigate the effects of instrument temperature and aperture on the maximum usable wavelength, as well as the effects of exozodiacal disk inclination and thickness on observational SNR. We then identify the spectral resolutions which maximize observational SNR subject to detector noise and the required starlight suppression levels for the detection of various potential biomarker molecules $(\mathrm{H} 2 \mathrm{O}, \mathrm{O} 2$, $\mathrm{CO} 2$, and $\mathrm{CH} 4)$.
\end{abstract}

Keywords: coronagraphy; exoplanets; high resolution spectroscopy; exozodiacal background; exoEarths; HDC; exozodi

\section{INTRODUCTION}

Directly imaging Earth-like exoplanets, or exoEarths, is a massive challenge. Earth-like planets present contrast levels of $\sim 10^{-10}$ with respect to their parent stars, and orbit at sub-arcsecond separations. That being said, studying these planets comes with enormous scientific discovery potential, including the possibility of finding life elsewhere in the universe. Design efforts for $10^{-10}$ coronagraphs and starshades are underway, ${ }^{1-6}$ but there are still many problems to solve in their development. Among these problems are concerns about the viability of suppressing starlight to better than $10^{-10}$ stably over a large bandpass and small enough working angles to image the systems under consideration. By combining a high-contrast coronagraph with a high-resolution spectrograph in a technique known as high-dispersion coronagraphy (HDC), some of these obstacles can be mitigated. For example, by using HDC, the starlight suppression requirements for the telescope can be reduced due to HDC's higher tolerance of contaminating photons. ${ }^{7}$

Two factors which have heretofore not been fully quantified with regards to HDC are the thermal and exozodiacal backgrounds which will be present in any observation of an exoEarth. Thermal background is potentially important because many of the strongest spectral lines for potential biosignature gases are in the near-infrared, and the telescope may need to be cooled to observe them. This cooling may increase the cost of the mission, rendering observing those lines an unattractive prospect.

Exozodiacal dust is another background source which is not entirely characterized at present. Exozodi presents as an extended background in the observed systems, potentially washing out imaging and spectral observations

Further author information: (Send correspondence to Carl T. Coker)

Carl T. Coker: E-mail: carl.t.coker@jpl.nasa.gov, Telephone: 18183540104

Ji Wang: E-mail: ji.wang@caltech.edu, Telephone: 16263954981

Stuart Shaklan: E-mail: stuart.b.shaklan@jpl.nasa.gov, Telephone: 18183540105

Space Telescopes and Instrumentation 2018: Optical, Infrared, and Millimeter Wave, edited by Makenzie Lystrup,

Howard A. MacEwen, Giovanni G. Fazio, Proc. of SPIE Vol. 10698, 106985G

(C) 2018 SPIE · CCC code: 0277-786X/18/\$18 - doi: 10.1117/12.2313695

Proc. of SPIE Vol. 10698 106985G-1 
of planets in those systems. Although there are efforts underway to observe dust disks around Sun-like stars, ${ }^{8,9}$ the average amount of dust and its spectral characteristics are not yet known. In the absence of hard data, efforts have been made to determine the effects of exozodiacal dust on exoEarth observations, ${ }^{10}$ although these works did not assume an HDC instrument. Also unknown are the effects of exozodiacal background on the design parameters of the instrument, particularly the spectral resolution which maximizes signal-to-noise.

In this paper, we investigate the effects of thermal and exozodiacal background on observations of exoEarths with HDC instruments for telescopes with apertures between 1 and $15 \mathrm{~m}$. We simulate the thermal background of the instrument and a scaled Solar System zodiacal model to look at the maximum tolerable levels of both on observations of various spectral lines of potential biosignature gases, as well as how increasing exozodiacal contamination affects the desired spectral resolution of the spectrograph. We do this for observations of an Earth-like planet at a distance of $5 \mathrm{pc}$.

In Section 2, we discuss the design of our models. In Section 3, we present our results on the effects of thermal background on our simulated exoEarth observations. In Section 4, we do the same for exozodiacal background. In Section 5, we investigate the effects of exozodiacal background on the optimal spectral resolution for the observations, while in Section 6, we present a brief discussion and conclusion.

\section{SIMULATION DESIGN}

\subsection{Cross-Correlation Function SNR}

Each HDC simulation results in a cross-correlation function $(\mathrm{CCF})$ of the simulated spectra with our template spectrum. We repeat the HDC simulation 100 times for each set of parameters, recording the maximum CCF value within one spectral resolution element of the planet radial velocity for each run. Running the simulation 100 times results in a distribution of maximum CCF values. We then calculate the 16th and 84th percentiles of this distribution; half the distance between the two values corresponds to $1 \sigma$ for the distribution. This way of defining $1 \sigma$ is robust against outliers. The maximum CCF value divided by $1 \sigma$ gives the CCF SNR. We take the median of the 100 CCF SNRs so calculated as our final CCF SNR for each simulated observation.

\subsection{Model Atmosphere}

We generate the spectrum of an Earth-like exoplanet using Renyu Hu's atmospheric chemistry and radiative transfer model ${ }^{11-14}$ which calculates the abundance of each molecular species with altitude, including photochemical and disequilibrium processes. The disk-reflected light spectrum is calculated using eighth-order Gaussian integration and the $\delta$-2-stream approximation. We include the opacities of four potential biosignature gases (water, oxygen, carbon dioxide, and methane), calculating the planetary flux at a spectral resolution of $R=\lambda / \Delta \lambda=500,000$. This resolution is chosen to well resolve the individual spectral lines of the species under consideration. The final model atmosphere has been compared with mid-latitude terrestrial measurements and closely resembles the present-day Earth. ${ }^{11}$

As the Earth's disk-averaged spectrum cannot be reproduced by a single surface type or cloud deck, ${ }^{15-17}$ we use a combination of three different model spectra. First is a cloud-free surface with a low albedo representing the ocean and ice-free landmass, second a highly reflective cloud layer at an altitude of $\sim 4 \mathrm{~km}$ representing cumulus clouds, and finally a high cloud layer at an altitude of $\sim 12 \mathrm{~km}$ representing cirrus clouds. We average the three models together with equal weight to mimic the present day Earth, which has a typical cloud cover of roughly two thirds. ${ }^{18}$ The resulting spectrum is generally consistent with Earthshine experiments ${ }^{15,16}$ and EPOXI measurements. ${ }^{17}$ Figure 1 shows our model spectrum between 0.5 and $2.5 \mu \mathrm{m}$ at $R=500,000,1,000$, and 100 .

\subsection{Maximum Wavelength vs. Inner Working Angle}

One of the prime considerations for any astronomical observation is the wavelengths at which to observe, For spectra of exoEarths, the primary driver is the location of the spectral lines of the assumed gases that make up the planet's atmosphere. For an Earth-like planet, the majority of these lines are in the near-infrared, although there are also some strong lines/features towards the red end of the visible spectrum, as well as ozone absorption in the UV. Further complicating the picture for a coronagraphic or starshade mission is that the bandpasses 


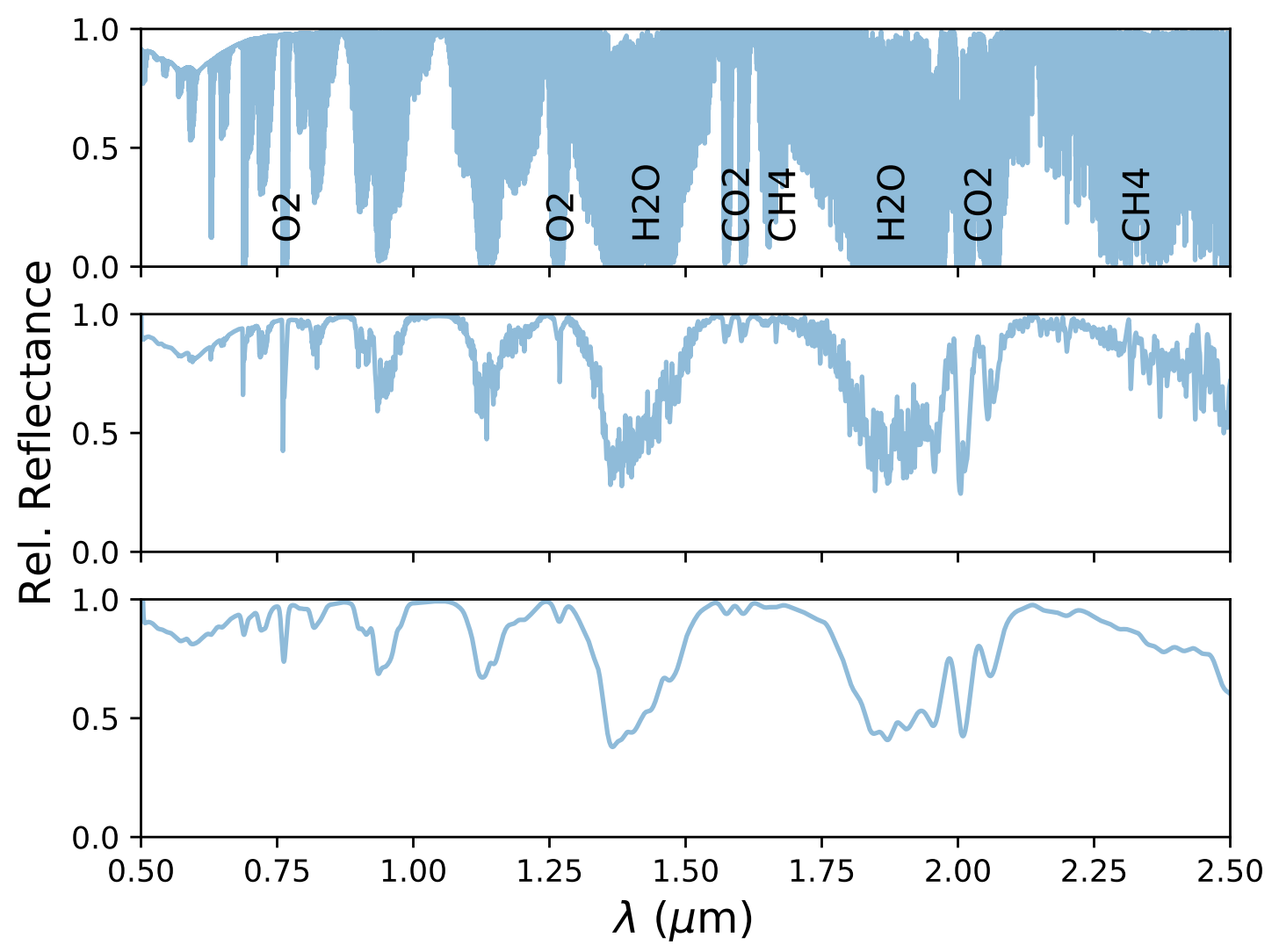

Figure 1. Model spectrum of an Earth-like exoplanet with water, oxygen, carbon dioxide, and methane included. This model is the average of a low-albedo no-cloud, reflective mid-level cloud, and high-level cloud model. Prominent spectral features are labeled; the vast majority of unlabeled bands are due to water. The slight dropoff in the visible range is due to ozone. The top panel shows $R=\lambda / \Delta \lambda=500,000$, the middle panel shows $R=1,000$, and the bottom panel shows $R=100$. 

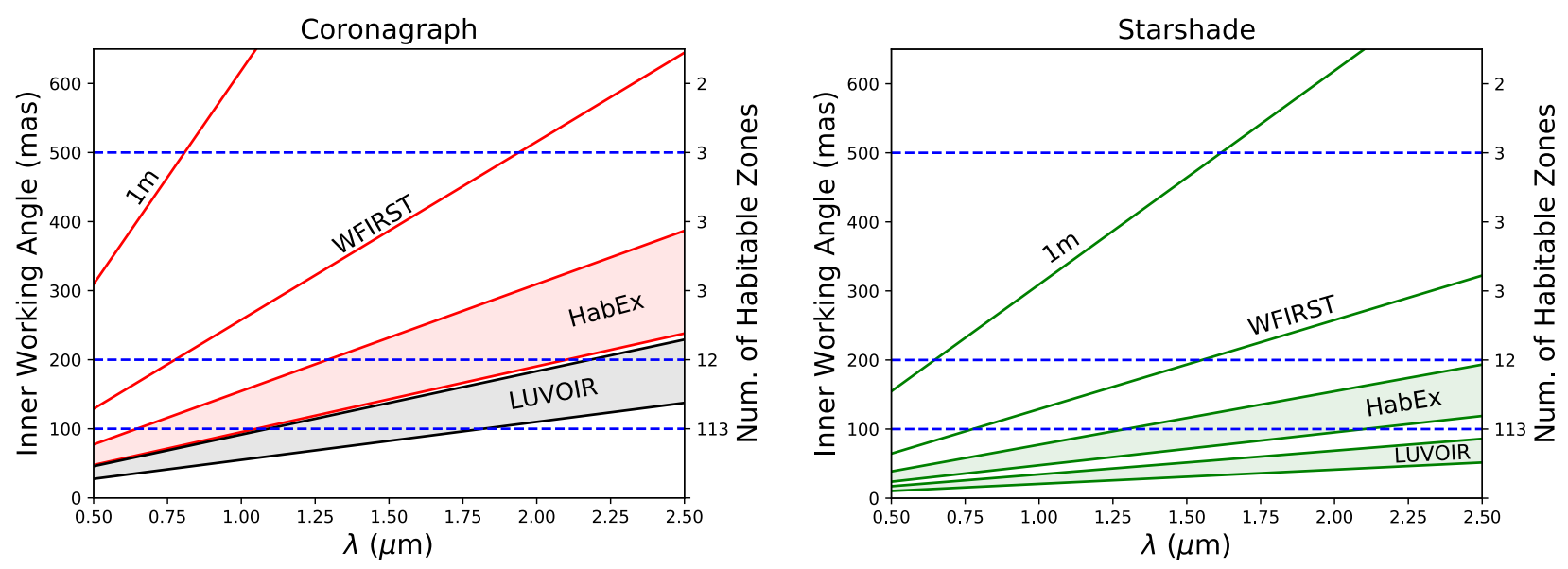

Figure 2. Inner working angle (IWA) vs. wavelength for a variety of telescope apertures. The left panel shows coronagraphic instruments, assuming an IWA of $3 \lambda / D$ for off-axis telescopes and WFIRST (red lines/regions), and $4 \lambda / D$ for on-axis telescopes (black lines/regions). WFIRST uses $3 \lambda / D$ in this work as that is the current design IWA for the WFIRST coronagraph. ${ }^{22}$ The right panel shows starshade designs, all of which have an IWA of $1.5 \lambda / D$. The dashed blue lines show the Earth-Sun separation at 2, 5, and 10 pc. The right axis shows the number of star systems in Exocat with an Earth-equivalent insolation distance greater than or equal to the IWA on the left axis, as a proxy for the number of habitable zones which are observable using a given instrument.

which can be used by those instruments for a single measurement are rather narrow (no more than $\sim 20 \%$ bandpass), as diffraction control at the necessary precision is highly chromatic. The spectral resolution used is another consideration, as some features may simply wash out entirely at low resolution. ${ }^{19}$

Figure 2 shows inner working angles (IWA) for a variety of aperture sizes and telescope concepts as a function of wavelength, using both coronagraphs and starshades. For the potential number of habitable zones that these instruments could probe, we use the Earth-equivalent insolation separation from the ExoCat catalog of nearby systems ${ }^{20}$ as a proxy. We removed all giant and subgiant stars from our estimate, as planets around these systems which were once habitable are unlikely to be so now. Of note is that to be very useful for observing potentially habitable planets, an instrument must have an IWA below $\sim 150-200$ mas; indeed, there are just three systems with an Earth-equivalent insolation distance greater than 300 mas: $\alpha$ Cen A and B and Procyon A (which is itself unlikely to have any habitable planets). For a coronagraphic instrument, this has the potential to severely restrict the maximum usable wavelength; only the LUVOIR ${ }^{21}$ concepts have a large enough aperture to be able to observe beyond $2 \mu \mathrm{m}$ using one. For starshade missions, the problem is greatly reduced by the smaller IWA.

Even with this challenge, however, it may be worth observing at wavelengths beyond $2 \mu \mathrm{m}$. For Earth-like planets, this would be driven by the strong carbon dioxide and methane bands present from $2-2.5 \mu \mathrm{m}$; as Figure 1 shows, they are far more visible at lower resolution than the carbon dioxide and methane lines at 1.6 and $1.75 \mu \mathrm{m}$, respectively. Therefore, we consider the effects of thermal and exozodiacal background on these lines to determine if observing them is feasible for current exoEarth observatory concepts. The potential very low temperature requirement for observing these lines is a concern, as cryogenic cooling would increase the cost of the mission and possibly shorten the mission lifetime.

\section{THERMAL BACKGROUND}

For infrared observatories, the thermal radiation from the telescope optics and instrument can be a major problem necessitating that the entire instrument be cooled. In the near-infrared for typical telescope operating temperatures, this problem becomes worse with increasing wavelength, as the telescope is operating on the exponential decay tail of the Planck distribution. As such, it is important to quantify the effect of the instrument's thermal background on its observations to ensure that it can observe the most important spectral lines for its 

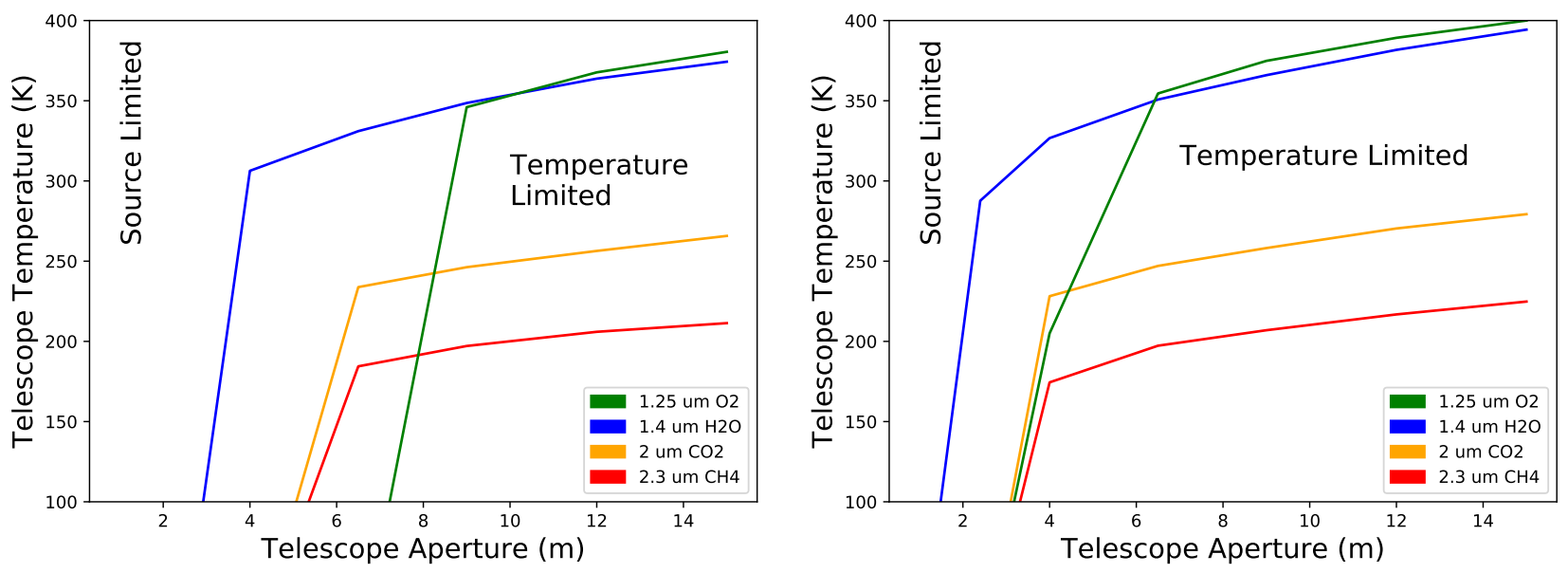

Figure 3. Maximum allowable temperature for observing the given spectral lines as a function of aperture. Red is the $2.3 \mu \mathrm{m}$ methane line, orange the $2.0 \mu \mathrm{m}$ carbon dioxide complex, blue the water band centered at $1.4 \mu \mathrm{m}$, and green the narrow oxygen band at $1.25 \mu \mathrm{m}$. We assume the Earth-Sun system at $d=5 \mathrm{pc}$ and $i=60^{\circ}$ for the purposes of these simulations. Total integration is 100 hours in the left panel and 400 hours in the right panel, with zero detector noise. For lines redder than $2 \mu \mathrm{m}$, more intensive cooling is required, but the telescope/instrument thermal background is otherwise not a limiting factor on observations.

observations - in this particular case, lines for biomarker molecules such as water, carbon dioxide, oxygen, and methane. In our simulations, we chose wavelength ranges which just covered the lines/bands in question.

Figure 3 shows the maximum temperature which allows a CCF SNR of 5 as a function of aperture for a selection of spectral lines from water, carbon dioxide, oxygen, and methane, given an exposure time of 100 hours in the left panel and 400 hours in the right panel. These exposure times correspond to plausible maximum exposure times for the LUVOIR and HabEx ${ }^{23}$ mission concepts, respectively. The LUVOIR concept is able to devote less time per target than the HabEx concept because it is conceived as a general purpose astronomical observatory, and is thus not dedicated mostly to searching for and characterizing exoplanets.

In all cases, we model observations of the Earth-Sun system at $5 \mathrm{pc}$, as our goal is to provide a baseline for observing potentially life-bearing planets. $\mathrm{R}=1,000$ was chosen as a middle ground between previous results for the LUVOIR and HabEx concepts. ${ }^{7}$ We did not include the effects of detector noise in these simulations because they would obscure the behavior we were attempting to characterize. To calculate the thermal background, we assume 10 gold surfaces, calculating the reflectance from refractive index data. ${ }^{24,25}$ For each molecule under consideration, we chose one representative spectral line/band, with the goal being to cover the near-infrared spectrum to $2.5 \mu \mathrm{m}$. Other lines of these species may be easier to detect than shown. We included an exozodiacal disk corresponding to the Solar System at an inclination of $60^{\circ}$ (see Section 4 for more details on this model).

A full JWST-style sunshade is not necessary to observe any of the lines under consideration, however; if the $2.3 \mu \mathrm{m}$ methane line is a target, the telescope temperature should be maintained under $200 \mathrm{~K}$. Lines blueward of $2 \mu \mathrm{m}$ do not require any sort of special cooling for the HabEx or LUVOIR concepts. The steep drops in the maximum temperature curves at smaller apertures represent the fact the line was not detected in the simulations at those apertures, even with zero thermal background, meaning that thermal background is not the limiting factor in those observations.

\section{EXOZODIACAL BACKGROUND}

One of the great unknowns in the search for exoEarths is the level of exozodiacal dust, and thus exozodiacal background, that will be encountered in those systems. The more dust is present, the harder it will be to detect planets in those systems. This is especially important because only a small minority of systems will be face-on, and the higher the inclination to our line of sight, the more exozodiacal dust will affect our observations. To 

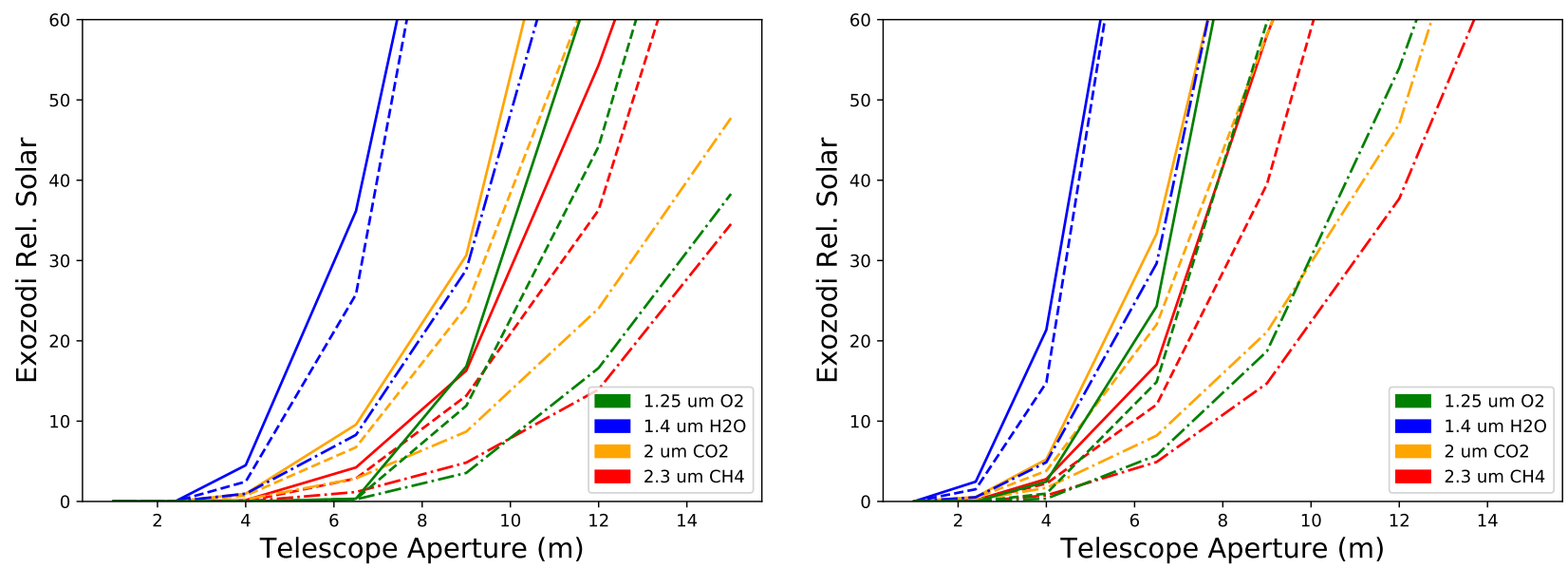

Figure 4. Maximum level of exozodi for observing the given spectral lines as a function of aperture. Red is the $2.3 \mu \mathrm{m}$ methane line, orange the $2.0 \mu \mathrm{m}$ carbon dioxide complex, blue the water band centered at $1.4 \mu \mathrm{m}$, and green the narrow oxygen band at $1.25 \mu \mathrm{m}$. We assume the Earth-Sun system at $d=5 \mathrm{pc}$ and an instrument temperature of $100 \mathrm{~K}$ for the purposes of these simulations. Total integration is 100 hours in the left panel and 400 hours in the right panel, with zero detector noise. The solid, dashed, and dot-dashed lines show the simulated system at $i=0^{\circ}, 60^{\circ}$, and $90^{\circ}$, respectively. Moving from a face-on to an edge-on zodiacal disk is a factor of $\sim 3-5$ effect in CCF SNR.

help model and account for this effect, we used the Haystacks model of the Solar System zodiacal disk ${ }^{26}$ and scaled it to the desired exozodi level.

Figure 4 shows how changing the exozodiacal background level and inclination affect HDC observations of exoEarths. The lines on each figure show the maximum exozodiacal background which allows a CCF SNR of 5 as a function of aperture for each considered spectral line. These spectral features are the same ones assumed in the plots in Section 3. Spectral resolution is set to 1,000, and no detector noise is included, for the reasons given in Section 3. We assume a telescope temperature of $100 \mathrm{~K}$. We model inclinations of $0^{\circ}, 60^{\circ}$, and $90^{\circ}$, covering the full range to examine how observability of biosignature gases changes with viewing angle. We find that the CCF SNR decreases by a factor of $\sim 3-5$ between the face-on and edge-on cases, depending on spectral line, as shown by the difference between the solid and dot-dashed lines in Figure 4.

The larger the telescope aperture, the more robust it is against exozodiacal contamination. This occurs for two reasons: first, there is more signal from the planet due to the larger aperture, and second, that the PSF shrinks as $D^{2}$, meaning there is less exozodi contamination of the spatially unresolved planet signal in larger telescopes. PSF area is the dominant effect in our simulations, driving the shape of the curves in Figure 4. Also of note is that exozodiacal background can have a strong effect on which lines are detectable - the $1.25 \mu \mathrm{m}$ oxygen line in particular is difficult to detect using smaller apertures, and even moderate exozodi can render it invisible to the CCF technique if long integrations are not used. This is because the line is narrow and has low spectral information. Increasing the spectral resolution would increase the CCF SNR as well (see Section 5), raising the maximum allowable exozodi level.

\section{OPTIMAL SPECTRAL RESOLUTION}

Although HDC has many benefits, it is not without limits. Spectral resolution cannot be increased infinitely - even given zero-noise detectors, once the observed spectrum is fully resolved no further information can be extracted. Additionally, at very high spectral resolutions less than one photon per resolution element may be expected over the course of the observation, and so it is pointless to increase the resolution further. For a $4 \mathrm{~m}$ HabEx concept, for example, this occurs at $R \gtrsim 50,000$ for a 400 hour integration. ${ }^{7}$ Detector noise also increases with spectral resolution as read noise and dark current are per pixel noise sources. Meanwhile, speckle noise can mimic low resolution features. ${ }^{7}$ This means that the spectral resolution for an HDC instrument which maximizes 
SNR is the one which balances the effects of detector and speckle noise in the absence of other backgrounds. Here we test whether the addition of exozodiacal background would influence the optimal spectral resolution instrument with its additional associated photon noise and spectral features.

Figure 5 shows the signal-to-noise achieved as a function of spectral resolution and starlight suppression for the $6.5 \mathrm{~m}$ HabEx concept on a $i=60^{\circ}$ system with exozodi levels of 1, 5, 10, and 20 times the Solar System. To simulate a more realistic mission, we chose a bandwidth of $20 \%$ stretching from 1.4 to $1.7 \mu \mathrm{m}$. This bandpass was picked to cover a particularly rich region of the infrared spectrum which contains lines from water, carbon dioxide, and methane, although it does miss oxygen. We then added in detector noise in the form of read noise at a level of $2.0 \mathrm{e}^{-} \mathrm{read}^{-1} \mathrm{pix}^{-1}$ and dark current at $0.002 \mathrm{e}^{-} \mathrm{s}^{-1} \mathrm{pix}^{-1}$; we assumed three pixels per resolution element and one read per hour of integration. Exposure time is 400 hours for all simulations. We assume an instrument temperature of $260 \mathrm{~K}$, which matches the target for the HabEx concept. ${ }^{23}$ Such a temperature is easily achieved for space telescope cooling systems, and does not represent any extra investment in telescope systems for this region of the IR spectrum, nor does it impede observations in this wavelength range, as shown in Section 3. Figure 6 shows the same for a $12 \mathrm{~m}$ LUVOIR concept.

Increasing the exozodi level has only a weak effect on the ideal spectral resolution of the instrument, increasing by a factor of $\sim 2$ for a 20 -fold increase in exozodi. This is likely because in the near-infrared region of the spectrum, zodiacal dust lacks narrow absorption lines and forms a smooth low-resolution continuum background which is passed over by the CCF. As such, the only effect of increased zodi is to decrease the achieved SNR due to the additional photon noise. Therefore, the expected level of exozodi should not influence the design of the spectrograph itself unless very high levels of exozodiacal dust are expected, although it could place more stringent requirements on starlight suppression.

\section{CONCLUSIONS}

In this paper, we studied the effects of thermal and exozodiacal backgrounds on HDC observations of exoEarths. We determined maximum allowable temperatures and exozodi levels for observing representative spectral features of four potential biosignature gases (water, oxygen, carbon dioxide, and methane) across a range of telescope apertures for the Earth-Sun system at 5 pc. We also investigated the effect of exozodiacal background on the optimal spectral resolution for the HabEx and LUVOIR concepts.

We find that telescope/instrument thermal background is not a large concern for most near-infrared spectral lines, only being particularly relevant for lines with $\lambda \gtrsim 2 \mu \mathrm{m}$. The amount of cooling necessary to observe those redder lines is not excessive, and does not require a cryogenic system with liquid coolant. The large inner working angle at long wavelengths quickly becomes more of a limitation than the thermal background.

Large aperture telescopes like the LUVOIR concept are relatively robust against exozodiacal contamination, able to detect all four species in non-edge-on systems at levels of zodiacal dust at least 15 times that of the Solar System in a 100-hour integration. The HabEx concept has more difficulty detecting molecules besides water at Earth-like concentrations, even with a longer 400-hour integration time, but is still able to tolerate exozodi levels up to $\sim 10$ times Solar in its $6.5 \mathrm{~m}$ aperture configuration. Exozodiacal background also has a weak effect on the optimal spectral resolution for HDC observations. A 20 zodi disk is best observed with a spectral resolution $\sim 2$ times higher than a Solar System-like disk. Moving to a larger aperture has no effect on the location of maximum SNR.

\section{ACKNOWLEDGMENTS}

We thank Renyu $\mathrm{Hu}$ for providing the atmospheric models used in this paper. This work was supported by an appointment to the NASA Postdoctoral Program at the Jet Propulsion Laboratory, administered by the Universities Space Research Association under contract with NASA. Copyright 2018 California Institute of Technology. Government Sponsorship acknowledged. All rights reserved. 

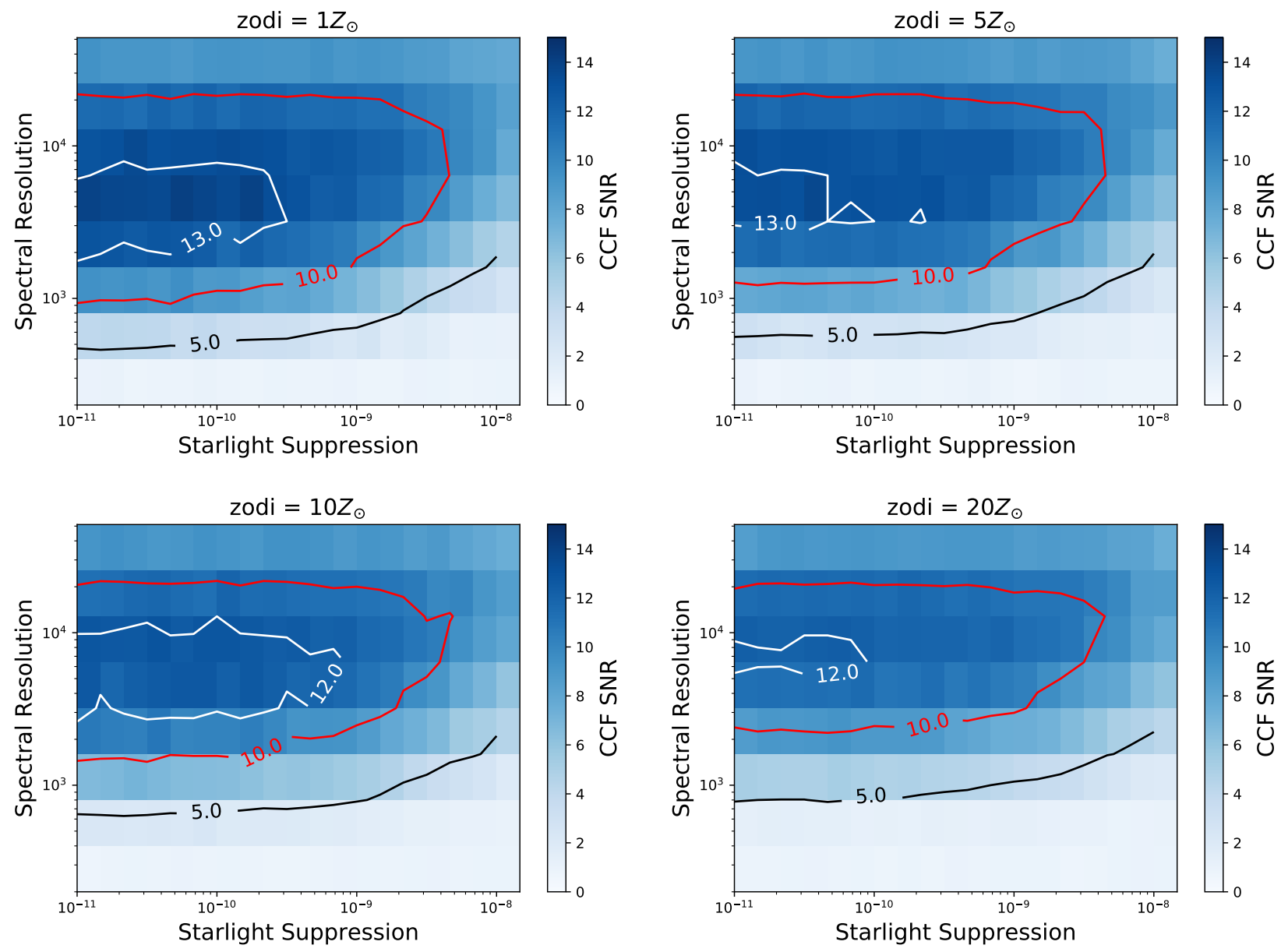

Figure 5. SNR achieved as a function of spectral resolution and starlight suppression for a $6.5 \mathrm{~m}$ HabEx concept on the Earth-Sun system at $d=5 \mathrm{pc}$ and $i=60^{\circ}$. From top left, the simulated exozodi level is $1,5,10$, and 20 times that of the Solar System. Increasing the exozodi level has only a weak effect on the desired spectral resolution. This is likely because it mostly forms a low-resolution continuum background in the near infrared. The fact that the maximum SNR is not seen at maximum starlight suppression is due to numerical noise. 

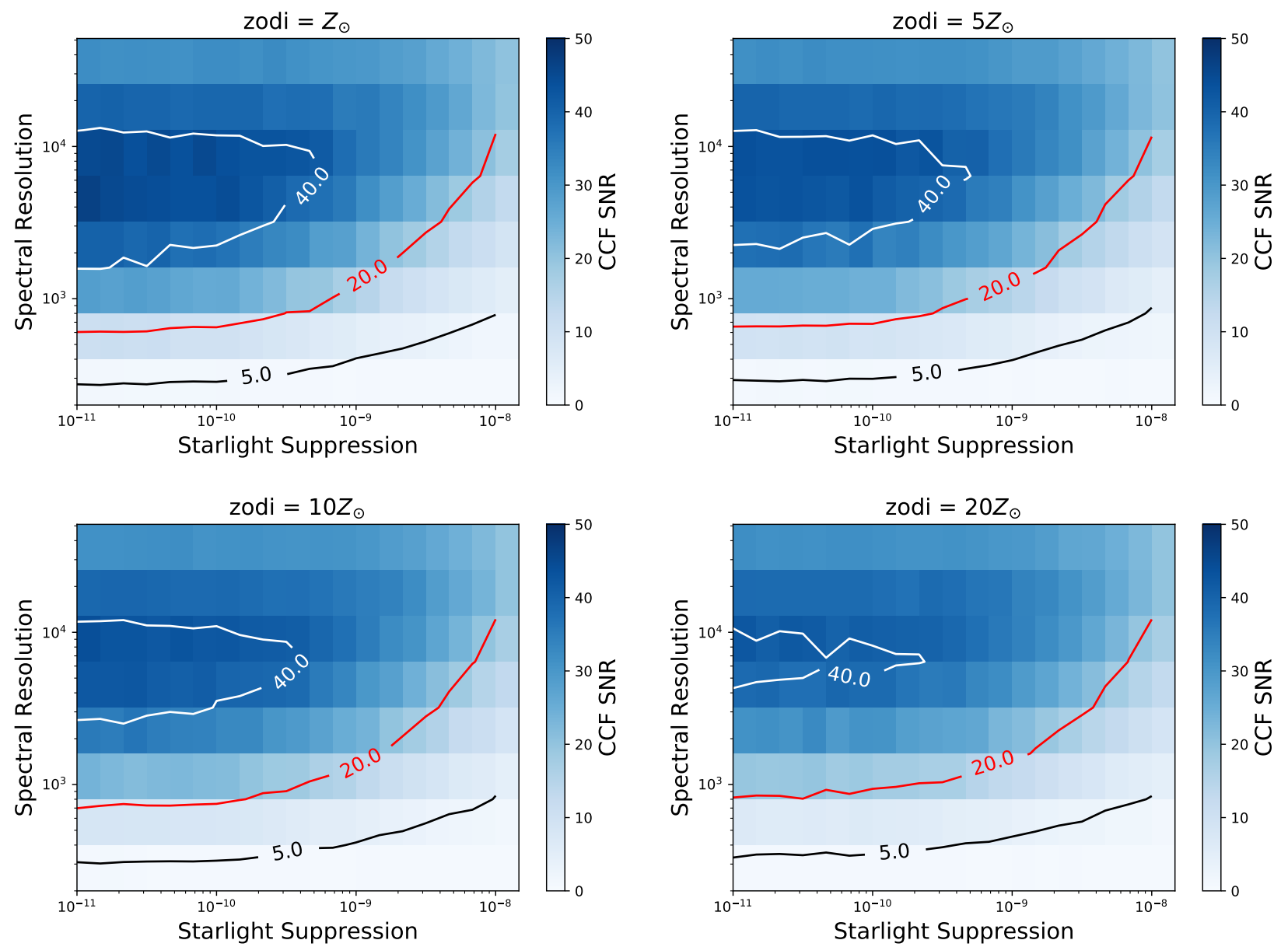

Figure 6. Same as Figure 5, but for a $12 \mathrm{~m}$ LUVOIR concept instead. The larger aperture does not change the optimal spectral resolution of the instrument. 


\section{REFERENCES}

[1] Turnbull, M. C., Glassman, T., Roberge, A., Cash, W., Noecker, C., Lo, A., Mason, B., Oakley, P., and Bally, J., "The Search for Habitable Worlds. 1. The Viability of a Starshade Mission", PASP, 124, 418 (2012)

[2] N'Diaye, M., Soummer, R., Pueyo, L., Carlotti, A., Stark, C. C., and Perrin, M. D., "Apodized Pupil Lyot Coronagraphs for Arbitrary Apertures. V. Hybrid Shaped Pupil Designs for Imaging Earth-like planets with Future Space Observatories", ApJ, 818, 163 (2016)

[3] Stark, C. C., Shaklan, S., Lisman, D., Cady, E., Savransky, D., Roberge, A., and Mandell, A. M., "Maximized exoEarth candidate yields for starshades", JATIS, 2, 041204 (2016)

[4] Zimmerman, N. T., N'Diaye, M., St. Laurent, K. E., Soummer, R., Pueyo, L., Stark, C. C., Sivaramakrishnan, A., Perrin, M., Vanderbei, R. J., Kasdin, N. J., Shaklan, S., and Carlotti, A., "Lyot coronagraph design study for large, segmented space telescope apertures", Proc. SPIE, 9904, 99041Y (2016)

[5] Mazoyer, J., Pueyo, L., N'Diaye, M., Fogarty, K., Zimmerman, N., Soummer, R., Shaklan, S., and Norman, C., "Active Correction of Aperture Discontinuities-Optimized Stroke Minimization. II. Optimization for Future Missions", ApJ, 155, 8 (2018)

[6] Ruane, G., Mawet, D., Mennesson, B., Jewell, J. B., and Shaklan, S. B., "Vortex coronagraphs for the Habitable Exoplanet Imaging Mission concept: theoretical performance and telescope requirements", JATIS, 4, 015004 (2018)

[7] Wang, J., Mawet, D., Ruane, G., Hu, R., and Benneke, B., "Observing Exoplanets with High Dispersion Coronagraphy. I. The Scientific Potential of Current and Next-generation Large Ground and Space Telescopes", AJ, 153, 183 (2017)

[8] Danchi, W., Bailey, V., Bryden, G., Defrère, Haniff, C., Hinz, P., Kennedy, G., Mennesson, B., MillanGabet, R., Rieke, G., Roberge, A., Serabyn, E., Skemer, A., Stapelfeldt, K., Weinberger, A., and Wyatt, M., "The LBTI hunt for observable signatures of terrestrial systems (HOSTS) survey: a key NASA science program on the road to exoplanet imaging missions", Proc. SPIE, 9146, 914607 (2014)

[9] Ertel, S., Defrère, Hinz, P., Mennesson, B., Kennedy, G. M., Danchi, W. C., Gelino, C., Hill, J. M., Hoffmann, W. F., Rieke, G., Shannon, A., Spalding, E., Stone, J. M., Vaz, A., Weinberger, A. J., Willems, P., Absil, O., Arbo, P., Bailey, V. P., Beichman, C., Bryden, G., Downey, E. C., Durney, O., Esposito, S., Gaspar, A., Grenz, P., Haniff, C. A., Leisenring, J. M., Marion, L., McMahon, T. J., Millan-Gabet, R., Montoya, M., Morzinski, K. M., Pinna, E., Power, J., Puglisi, A., Roberge, A., Serabyn, E., Skemer, A. J., Stapelfeldt, K., Su, Y. L., Vaitheeswaran, V., and Wyatt, M. C., "The HOSTS survey - Exozodiacal dust measurements for 30 stars", arXiv:1803.11265 (2018)

[10] Stark, C. C., Roberge, A., Mandell, A., and Robinson, T. D., "Maximizing the ExoEarth Candidate Yield from a Future Direct Imaging Mission", ApJ, 795, 122 (2014)

[11] Hu, R., Ehlmann, B. L., and Seager, S., "Theoretical Spectra of Terrestrial Exoplanet Surfaces", ApJ, 752, $7(2012)$

[12] Hu, R., Seager, S., and Bains, W., "Photochemistry in Terrestrial Exoplanet Atmospheres. I. Photochemistry Model and Benchmark Cases", ApJ, 761, 166 (2012)

[13] Hu, R., Seager, S., and Bains, W., "Photochemistry in Terrestrial Exoplanet Atmospheres. II. H2S and SO2 Photochemistry in Anoxic Atmospheres", ApJ, 769, 6 (2013)

[14] Hu, R., and Seager, S., "Photochemistry in Terrestrial Exoplanet Atmospheres. III. Photochemistry and Thermochemistry in Thick Atmospheres on Super Earths and Mini Neptunes", ApJ, 784, 63 (2014)

[15] Tinetti, G., Meadows, V. S., Crisp, D., Fong, W., Fishbein, E., Turnbull, M., and Bibring, J.-P., "Detectability of Planetary Characteristics in Disk-Averaged Spectra. I: The Earth Model", Astrobiology, 6, 34 (2006)

[16] Turnbull, M. C., Traub, W. A., Jucks, K. W., Woolf, N. J., Meyer, M. R., Gorlova, N., Skrutskie, M. F., and Wilson, J. C., "Spectrum of a Habitable World: Earthshine in the Near-Infrared", ApJ, 644, 551 (2006)

[17] Robinson, T. D., Meadows, V. S., Crisp, D., Deming, D., A'Hearn, M. F., Charbonneau, D., Livengood, T. A., Seager, S., Barry, R. K., Hearty, T., Hewagama, T., Lisse, C. M., McFadden, L. A., and Wellnitz, D. D., "Earth as an Extrasolar Planet: Earth Model Validation Using EPOXI Earth Observations", Astrobiology, 11, 393 (2011) 
[18] Stubenrauch, C. J., Rossow, W. B., Kinne, S., Ackerman, S., Cesana, G., Chepfer, H., Di Girolamo, L., Getzewich, B., Guignard, A., Heidinger, A., Maddux, B. C., Menzel, W. P., Minnis, P., Pearl, C., Platnick, S., Poulsen, C., Riedi, J., Sun-Mack, S., Walther, A., Winker, D., Zeng, S., and Zhao, G., "Assessment of Global Cloud Datasets from Satellites: Project and Database Initiated by the GEWEX Radiation Panel ", BAMS, 94, 1031-1049 (2013)

[19] Wang, J., Mawet, D., Ruane, G., Delorme, J.-R., Klimovich, N., and Hu, R., "Baseline requirements for detecting biosignatures with the HabEx and LUVOIR mission concepts", Proc. SPIE, 10400, 104000Z (2017)

[20] Turnbull, M. C., "ExoCat-1: The Nearby Stellar Systems Catalog for Exoplanet Imaging Missions", arXiv:1510.01731 (2015)

[21] Bolcar, M. R., Aloezos, S., Bly, V. T., Collins, C., Crooke, J., Dressing, C. D., Fantano, L., Feinberg, L. D., France, K., Gochar, G., Gong, Q., Hylan, J. E., Jones, A., Linares, I., Postman, M., Pueyo, L., Roberge, A., Sacks, L., Tompkins, S., and West, G., "The Large UV/Optical/Infrared Surveyor (LUVOIR): Decadal Mission concept design update", Proc. SPIE, 10398, 1039809 (2017)

[22] Poberezhskiy, I., Zhao, F., An, X., Balasubramanian, K., Belikov, R., Cady, E., Demers, R., Diaz, R., Gong, Q., Gordon, B., Goullioud, R., Greer, F., Guyon, O., Hoenk, M., Kasdin, N. J., Kern, B., Krist, J., Kuhnert, A., McElwain, M., Mennesson, B., Moody, D., Muller, R., Nemati, B., Patterson, K., Riggs, A. J., Ryan, D., Seo, B.-J., Shaklan, S., Sidick, E., Shi, F., Siegler, N., Soummer, R., Tang, H., Trauger, J., Wallace, J. K., Wang, X., White, V., Wilson, D., Yee, K., Zhou, H., and Zimmerman, N., "Technology development towards WFIRST-AFTA coronagraph", Proc. SPIE, 9143, 91430P (2014)

[23] Mennesson, B., Gaudi, S., Seager, S., Cahoy, K., Domagal-Goldman, S., Feinberg, L., Guyon, O., Kasdin, J., Marois, C., Mawet, D., Tamura, M., Mouillet, D., Prusti, T., Quirrenbach, A., Robinson, T., Rogers, L., Scowen, P., Somerville, R., Stapelfeldt, K., Stern, D., Still, M., Turnbull, M., Booth, J., Kiessling, A., Kuan, G., and Warfield, K., "The Habitable Exoplanet (HabEx) Imaging Mission: preliminary science drivers and technical requirements", Proc. SPIE, 9904, 99040L (2016)

[24] McPeak, K. M., Jayanti, S. V., Kress, S. J. P., Meyer, S., Iotti, S., Rossinelli, A., and Norris, D. J., "Plasmonic films can easily be better: Rules and recipes", ACS Photonics, 2, 326 (2015)

[25] Olmon, R. L., Slovick, B., Johnson, T. W., Shelton, D., Oh, S.-H., Boreman, G. D., and Raschke, M. B., "Optical dielectric function of gold", Phys Rev. B, 86, 235147 (2012)

[26] Roberge, A., Rizzo, M. J., Lincowski, A. P., Arney, G. N., Stark, C. C., Robinson, T. D., Snyder, G. F., Pueyo, L., Zimmerman, N. T., Jansen, T., Nesvold, E. R., Meadows, V. S., and Turnbull, M. C., "Finding the Needles in the Haystacks: High-fidelity Models of the Modern and Archean Solar System for Simulating Exoplanet Observations", PASP, 129, 124401 (2017) 